\title{
Adaptive Adjustment of Unit Tuning to Sound Localization Cues in Response to Monaural Occlusion in Developing Owl Optic Tectum
}

\author{
Joachim Mogdans and Eric I. Knudsen \\ Department of Neurobiology, Stanford University School of Medicine, Stanford, California 94305
}

Bimodal units in the barn owl's optic tectum are tuned to the location of auditory and visual stimuli, and are systematically organized according to their spatial tuning to form mutually aligned maps of auditory and visual space. Map alignment results from the fact that, normally, units are tuned to the values of interaural level difference (ILD) and interaural time difference (ITD) produced by a sound source at the location of their visual receptive fields (VRFs). Monaural occlusion alters the correspondence of ILD and ITD values with I0cations in space. We investigated the effect that raising owls with a chronic monaural occlusion has on the tuning of tectal units to ILD and ITD. Owls were monaurally occluded beginning at 1 month of age. The effects of monaural occlusion were assessed 2-4 months later by comparing the ILD and ITD tuning of units in monaurally occluded owls with the ILD and ITD tuning of units with equivalent VRFs in normal owls.

ILD and ITD tuning was shifted substantially and in the direction of the unoccluded ear (the adaptive direction) in owls raised with a monaural occlusion. In most tecta, the mapped representations of ILD and ITD were shifted systematically. In addition, in some tecta, monaural occlusion induced a change in the topography of the ILD map such that ILD tuning remained essentially constant at values near $\mathbf{0} \mathrm{dB}$ over abnormally large portions of the tectum. Across all recording sites, the average shift in ILD tuning was $9 \mathrm{~dB}$ ( $n=396)$ and the average shift in ITD tuning was $40 \mu \mathrm{sec}$ $(n=414)$. In four of five animals, the magnitude of the effect was not equivalent on the two sides of the brain, the adjustments being significantly larger and more systematic on the side ipsilateral to the occlusion. Such differences in the altered ILD and ITD maps on the two sides of the brain in individual animals indicate that, although a component of the adaptive adjustment might be due to regulation of the gain and phase response of the monaural signals early in the auditory pathway, a major component of the adjustment must occur at or beyond the level where the encoding of ILDs and ITDs for left and right space separates.

Auditory and visual space are represented topographically across the optic tectum (the avian homolog of the mammalian superior colliculus). Auditory-visual bimodal units are tuned to the lo-

\footnotetext{
Received Dec. 17, 1991; revised Mar. 24, 1992; accepted Mar. 31, 1992.

This work was supported by a stipend from the Wissenschaftsausschuss of NATO through the Deutscher Akademischer Austauschdienst and by NIH Grant R01 DC00155-12. We thank M. S. Brainard, D. E. Feldman, T. T. Takahashi, and $\mathbf{H}$. Wagner for reviewing the manuscript, and P. F. Knudsen for graphic art. Correspondence should be addressed to Joachim Mogdans, Department of Neurobiology, Fairchild D257, Stanford University School of Medicine, Stanford, CA 94305-5401.

Copyright (C) 1992 Society for Neuroscience $0270-6474 / 92 / 123473-12 \$ 05.00 / 0$
}

cation of sound sources and respond maximally when a source is located in a restricted "best area" (Knudsen, 1982; King and Palmer, 1983; Middlebrooks and Knudsen, 1984; King and Ilutchings, 1987). The auditory best areas of thesc units align closely with their visual receptive fields (VRFs). The units are organized within the tectum according to the location of their auditory and visual spatial fields to form a multimodal map of space.

In the barn owl, the auditory spatial tuning of tectal units is based primarily on tuning to interaural time difference (ITD) and interaural level difference (ILD) (Olsen et al., 1989). ITDs vary systematically with the azimuth of a sound source. Due to an asymmetry in the owl's external ears, ILDs for frequencies above $4 \mathrm{kHz}$ vary with sound source elevation. Thus, the auditory best areas are limited in azimuth primarily as a result of unit tuning to ITD and in elevation as a result of unit tuning to ILD.

Occluding one ear with a plug attenuates and delays sound reaching the eardrum of the occluded ear (Knudsen et al., 1984). As a conscquence, monaural occlusion causes a systematic shift in the values of ILD and ITD that correspond with each location in space and, therefore, an immediate systematic shift in the locations of unit best areas (Knudsen and Konishi, 1980). Nevertheless, in animals raised with one ear occluded, auditory best areas of tectal units are aligned with VRFs as long as the earplug remains in place (Knudsen, 1985; King et al., 1988). When an earplug is removed from owls that have adjusted to the earplug. auditory best areas shift away from VRFs toward the side of the previously unplugged ear (Knudsen, 1983, 1985); if the earplug is removed at a sufficiently early age, auditory best areas gradually realign with VRFs. Thus, during development experience shapes the auditory spatial tuning of tectal units so as to maintain the alignment of the auditory and visual maps of space.

In the present study, we investigated the underlying basis of the adaptive adjustment of auditory spatial fields in response to carly monaural occlusion. The tuning of tectal units to binaural localization cues was compared in owls raised with or without monaural plugs. Since we did not measure auditory spatial tuning directly, we defined adaptive adjustment as a shift in unit tuning to binaural cues that was in the direction appropriate to realign the auditory best area with the VRF. The results demonstrate that monaural occlusion during early life does indeed dramatically alter unit tuning to ILD and ITD in an adaptive fashion.

\section{Materials and Methods}

Data were collected from six barn owls (Tyto alba) raised with one ear occluded and 15 normal owls; data from eight of the normal owls have been presented previously (Olsen et al., 1989). Because the methods 
Figure 1. Unit tuning to sound localization cues, ILD and ITD, was shifted in the adaptive direction as a result of early monaural occlusion. The plots compare the ILD tuning and ITD tuning of units from a normal owl (shaded lines) with those of units from either a right- $(A$ and $B)$ or a left- $(C$ and $D)$ earplugged owl (solid lines). The tuning curves are from recording sites with VRFs at similar locations: normal owl, $0^{\circ},-10^{\circ}$; right-earplugged owl (owl R1), $0^{\circ},-8^{\circ}$; left-earplugged owl (owl L1), $0^{\circ},-9^{\circ} . A$ and $C$ compare ILD tuning; $B$ and $D$ compare ITD tuning. The $a s$ terisks indicate best ILD and best ITD. Best ILD was $2.4 \mathrm{~dB}$ left-ear-greater in the normal owl, $15.5 \mathrm{~dB}$ left-ear-greater in the right-earplugged owl, and $6.4 \mathrm{~dB}$ right-ear-greater in the left-earplugged owl. Best ITD was $2.7 \mu$ sec right-earleading in the normal owl, $48.2 \mu \mathrm{sec}$ left-ear-leading in the right-earplugged owl, and 53.6 $\mu \mathrm{scc}$ right-car-lcading in the left-earplugged owl. Negative response values indicate spike counts below spontaneous level. Maximum spike counts were 69 (ILD) and 120 (ITD) for the normal owl, 157 (ILD) and 104 (ITD) for the right-earplugged, and 77 (ILD) and 158 (ITD) for the left-earplugged owl.

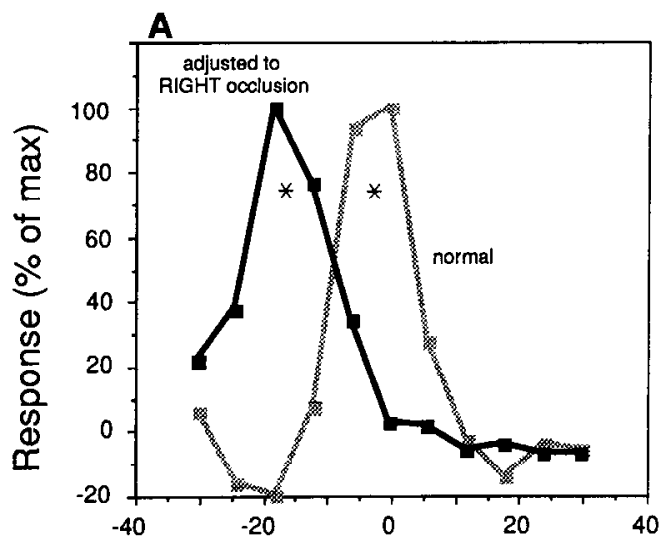

B
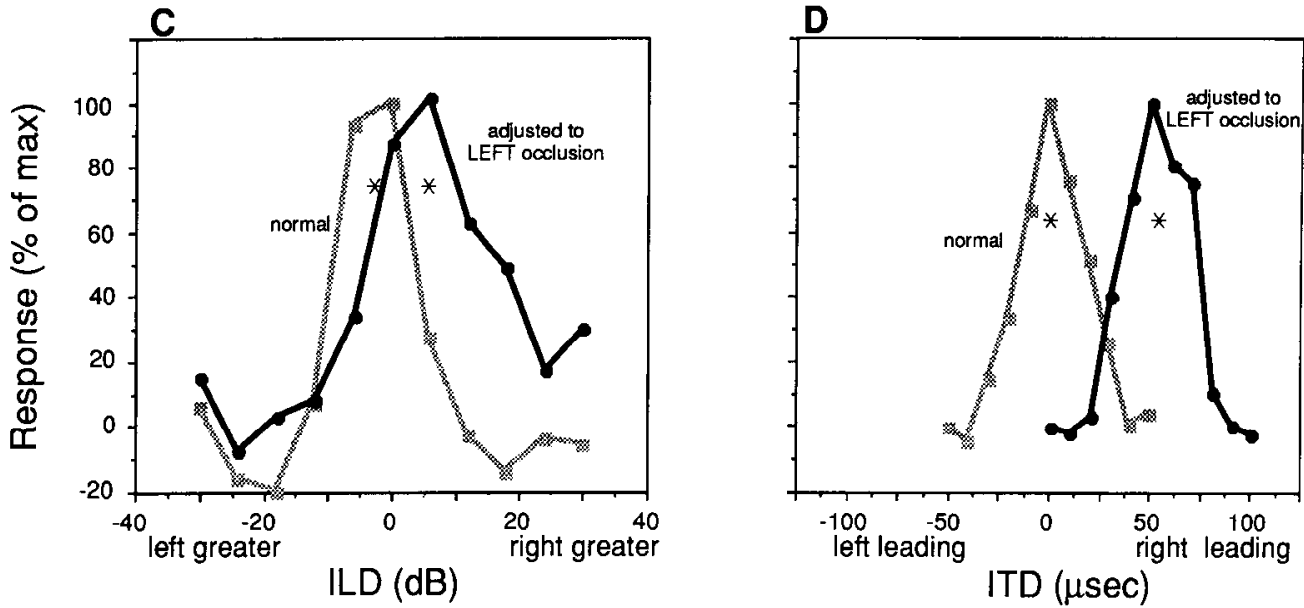

have been described in detail in previous reports (Knudsen et al., 1984, 1991; Knudsen, 1985; Olsen et al., 1989; Brainard et al., 1992), they will be outlined only briefly here.

Ear plugging. Barn owls were monaurally occluded at the age of 28$32 \mathrm{~d}$ by suturing a foam rubber plug (E.A.R. Cabot Corp.) into the external meatus while the animals were anesthetized with halothane and nitrous oxide. The right ear was plugged in three owls and the left ear was plugged in the other three. Owls were initially raised in boxes in groups of two. They were handled frequently to ensure rich auditory experience. At $60 \mathrm{~d}$ of age, when the birds began to fly, they were placed in a flight aviary. The birds were prepared for electrophysiological recording at 80-90 d of age. Before each experiment, the earplug was removed and the canal and eardrum were visually inspected for damage or accumulation of earwax. Earplugs were sutured back in place after the experiment.

No attempt was made to measure the acoustic properties of the earplugs, because implanting electrodes to record cochlear microphonics can damage the cochlea, which would render subsequently collected neurophysiological data uninterpretable. However, the effects of the identical earplugging procedure on adult owls were measured as part of another study (Knudsen et al., 1984). The effects are variable across individuals and are frequency dependent. Averaged over individuals, frequencies from 3 to $10 \mathrm{kHz}$ are attenuated by $17-53 \mathrm{~dB}$ and delayed by up to $80 \mu \mathrm{sec}$. Although the earplugs used here may have been this effective initially, their effectiveness probably decreased as the ear canals grew and the foam rubber plugs hardened. Nevertheless, the earplugs in all experimental birds remained complete and firmly in place until removed for an experiment.

Preparation. Owls were anesthetized with halothane and nitrous oxide to cement a head plate onto the skull and to make a small craniotomy over the region of the optic tectum. On the day of an experiment, the owl was anesthetized and tranquilized with intramuscular injections of ketamine $\mathrm{HCl}(15 \mathrm{mg} / \mathrm{kg})$ and diazepam $(5 \mathrm{mg} / \mathrm{kg})$. The owl was wrapped in a soft leather jacket and suspended in a prone position inside a sound isolation chamber equipped with earphones for dichotic stimulation.
The headplate was bolted to the stereotaxic apparatus, and the owl's head was positioned using retinal landmarks (the eyes are essentially stationary in the head). Visual field locations are reported in azimuth, indicating degrees left $(\mathrm{L})$ or right $(\mathrm{R})$ of the midsagittal plane, and elevation, indicating degrees above $(+)$ or below $(-)$ the visual (horizontal) plane. All angles were measured from the center of the owl's head. The properties of this double-pole coordinate system are described in detail in an earlier report (Knudsen, 1982). At the end of an experiment, chloramphenicol $(0.5 \%)$ was applied to the brain surface to prevent infections, the craniotomy was sealed with dental acrylic, incisions were infused with Xylocaine, and the ear was occluded with a fresh plug. After recovery from anesthesia $(1-2 \mathrm{~h})$, the owl was returned to its home cage.

Dichotic stimulation. Dichotic stimuli consisted of computer-generated noise or tone bursts, $50 \mathrm{msec}$ in duration, with rise/fall times of 0 msec for noise and $5 \mathrm{msec}$ for tones. Noise stimuli were highpass filtered digitally at $4 \mathrm{kHz}$ to ensure adequate isolation of the acoustic stimuli in the two ears. In the owl, acoustic isolation is limited by sound transmission through the interaural canal. Interaural attenuation of sound by the canal is frequency dependent, ranging from about $6 \mathrm{~dB}$ at $2 \mathrm{kHz}$ to $65 \mathrm{~dB}$ at $7 \mathrm{kHz}$ (Moiseff and Konishi, 1981); interaural attenuation is greater than $30 \mathrm{~dB}$ for frequencies above $4 \mathrm{kHz}$. ILDs exceding 30 $\mathrm{dB}$ were not delivered.

Stimuli were transduced by Knowles subminiature earphones (ED1914) coupled to damping assemblies (BF-1743). The earphones were centered in the ear canals and their ends aligned with a bony ridge that extends into the ear canal, to achieve equal insertion depth on both sides. The frequency response of each earphone was flat to within \pm 2 $\mathrm{dB}$ from 4 to $10 \mathrm{kHz}$. Interearphone differences were less than $3 \mu \mathrm{sec}$ and $2 \mathrm{~dB}$ between 4 and $12 \mathrm{kHz}$. The dynamic response of the earphones was linear to within $0.2 \mathrm{~dB}$ over a $45 \mathrm{~dB}$ range of input amplitudes. The amplitude in each earphone was controlled by a programmable attenuator, and time delays hetween the two channels were produced by computer-calculated shifts in the waveforms of the signal (Olsen et al., 1989). 
A

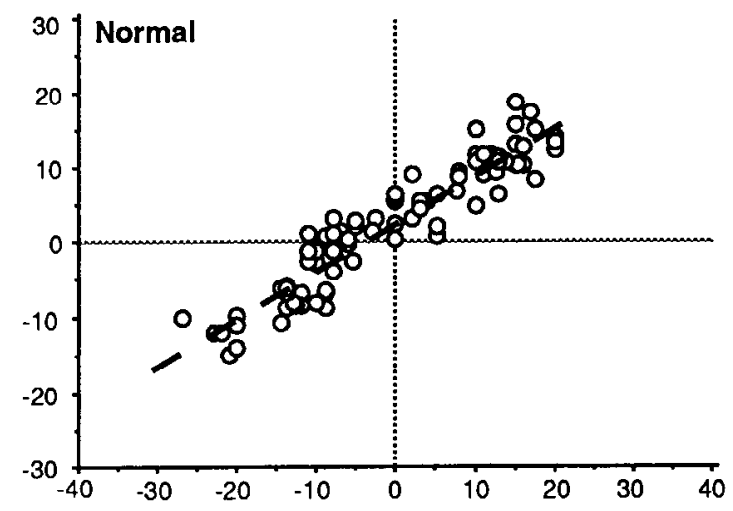

C
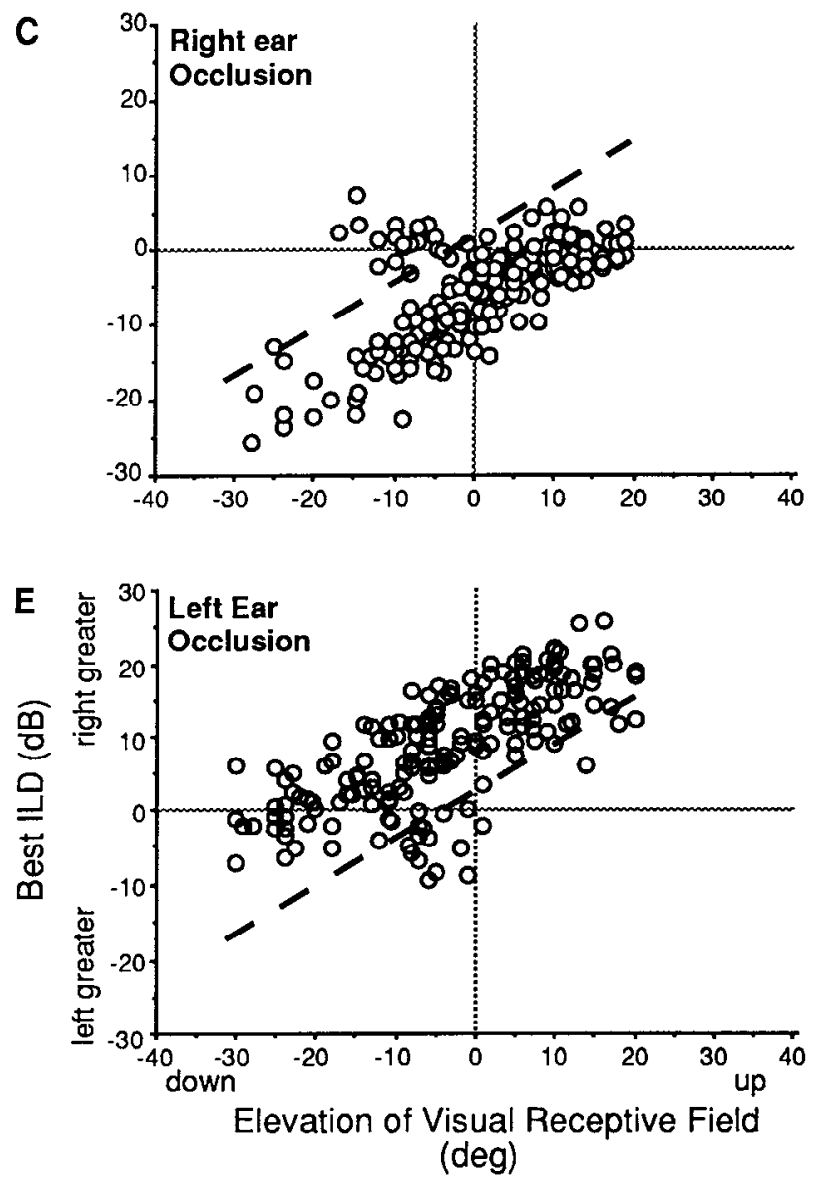
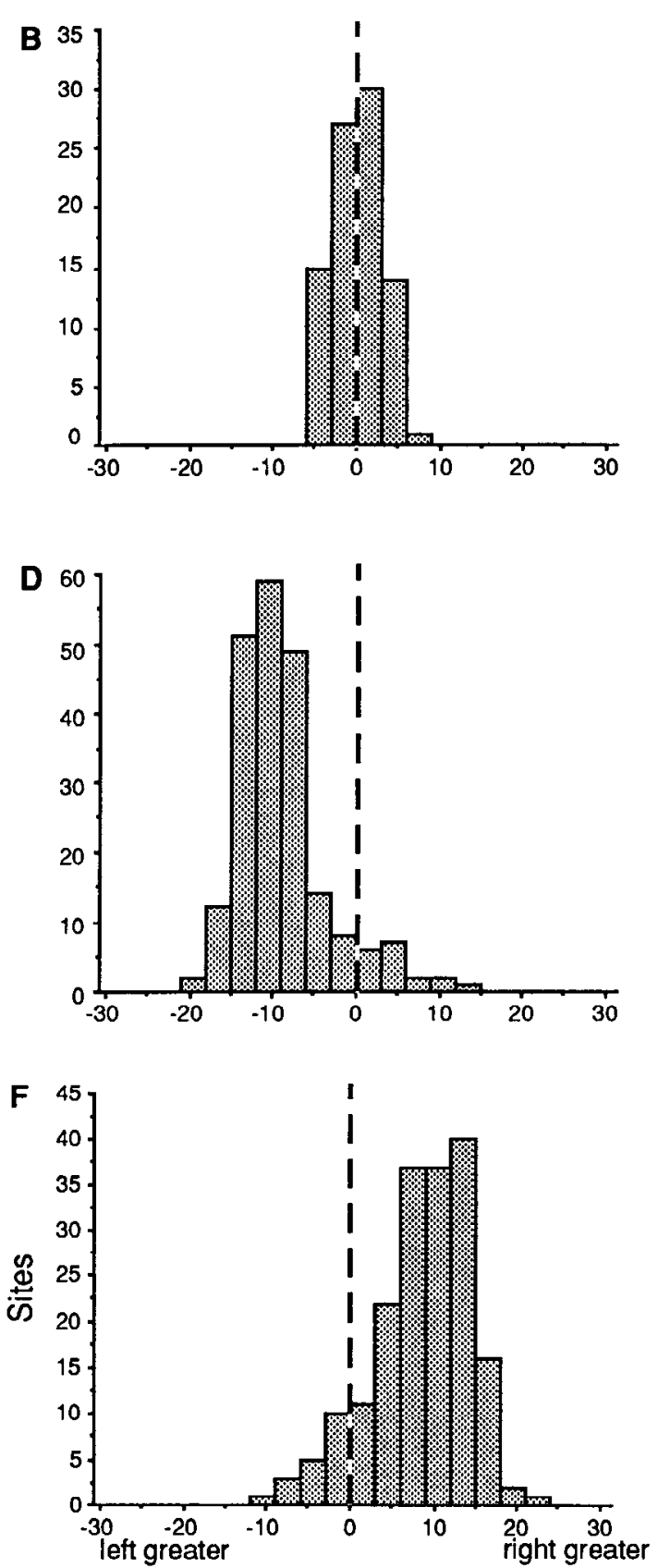

Difference from Normal Regression

(dB of ILD)

Figure 2. Relationship between best ILD and VRF elevation. Data are restricted to recording sites with VRFs located between $+20^{\circ}$ and $-30^{\circ}$ elevation and between $\mathrm{L} 20^{\circ}$ and R $20^{\circ}$ azimuth, and with closed ILD tuning curves (Table 1). A, Results of 88 recording sites from 15 normal owls. The regression line (broken line; $y=0.64 x+2.81 ; r^{2}-0.89$ ) was calculated to assess shifts in best ILDs measured in earplugged owls. $C$, Results of 212 recording sites from three right-earplugged owls. The regression for this data set $\left(y=0.48 x-6.0 ; r^{2}=0.43\right)$ was shifted toward left-eargreater ILDs. $E$, Results of 184 recording sites from three left-earplugged owls. The regression for this data set $\left(y=0.48 x+10.73 ; r^{2}=0.52\right)$ was shifted toward right-ear-greater ILDs. $B$. Differences between best ILDs measured in normal owls $(A)$ and best ILDs predicted from the normal regression line. $D$, Differences between best ILDs measured in right-earplugged owls $(C)$ and best ILDs predicted from the normal regression line (mean, $8.9 \mathrm{~dB}$ left-ear-greater; significantly different from $0, p<0.001) . F$, Differences between Best ILDs measured in left-earplugged owls $(E$ ) and best ILD predicted from the normal regression line (mean, $8.5 \mathrm{~dB}$ right-ear-greater; significantly different from $0, p<0.001$ ). The data for each owl are plotted separately in Figures 3 and 4.

Visual stimulation. Visual stimuli consisted of bars and spots produced by an ophthalmoscope that were projected onto a translucent hemisphere placed in front of the owl with the head of the owl at the center of the hemisphere. VRFs were defined as locations on the hemisphere where a visual stimulus produced an increase in a unit's discharge rate.

Neurophysiological recordings. A tungsten microelectrode was ad- vanced by a microdrive through the forebrain while unit activity was monitored on an oscilloscope and audio monitor. A characteristic bursting pattern of spontaneous activity signaled the entry of the electrode into the superficial layers of the optic tectum. The position of the electrode in the tectum was determined by measuring the location of the VRF and referring to the map of visual space in the optic tectum described by Knudsen (1982). Because of the curvature of the optic tectum, 


\section{RIGHT EAR OCCLUSION}
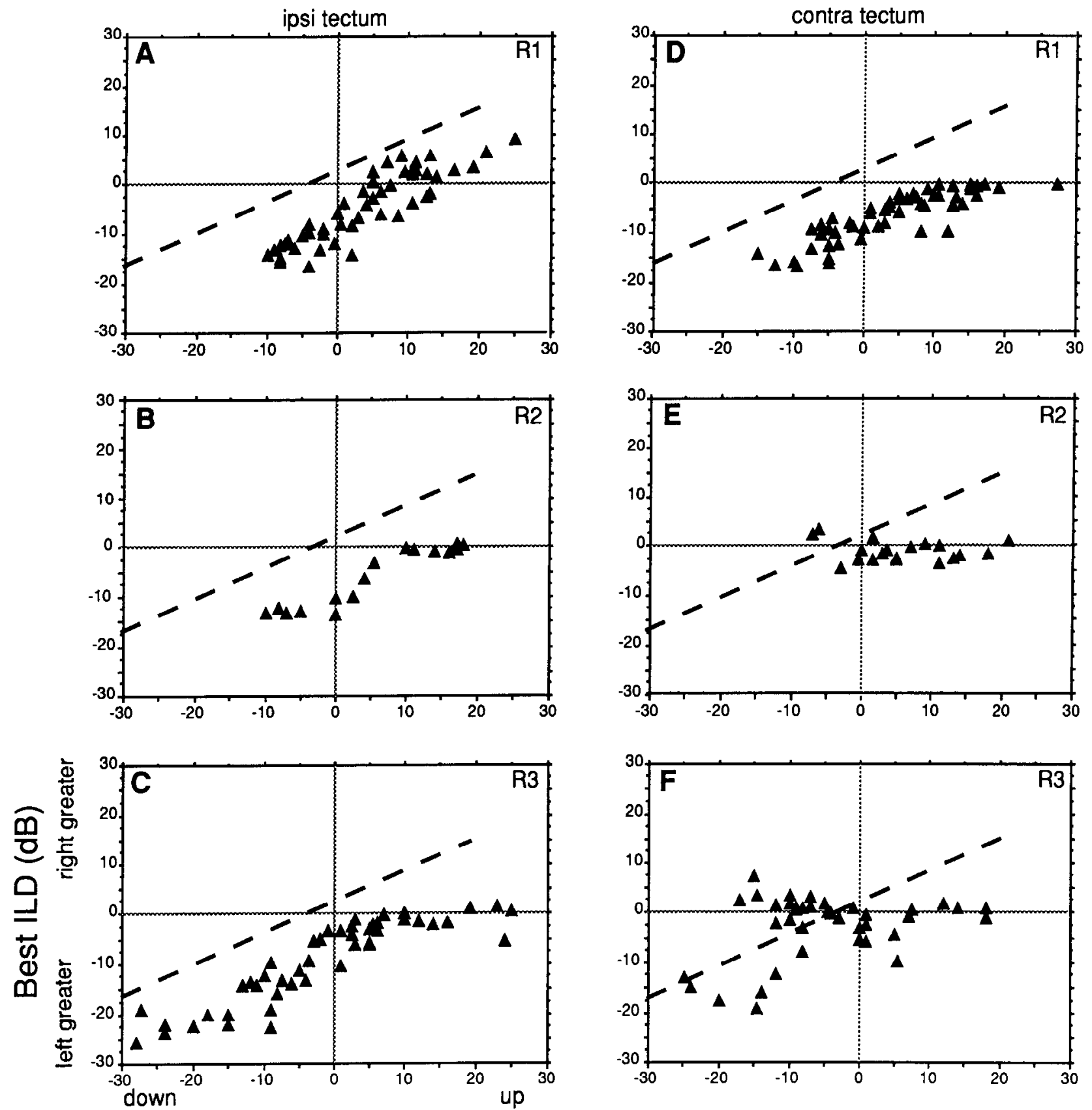

\section{Elevation of Visual Receptive Field (deg)}

Figure 3. Relationship between best ILD and VRF elevation in individual tecta of right-earplugged owls. $A-C$, Best ILDs in the tecta ipsilateral to the occluded ear. $D-F$, Best ILDs in the tecta contralateral to the occluded ear. Recording sites with VRFs located above $+20^{\circ}$ elevation are included in the graphs, but were not included in statistical analysis. For comparison, the regression line calculated for normal owls is shown as a broken line in each plot.

the electrode passed through the superficial layers twice: dorsally when entering and ventrally when exiting the nucleus. Action potentials from single units and multiple units were recorded extracellularly. There was no apparent difference in the ILD and ITD tuning of single- and multiple-unit recordings. Therefore, the data from all recording sites were combined. Many recordings sites in the deep layers of the optic tectum $(n=141)$ did not respond to visual stimulation. The depths of these recording sites were measured relative to the entry and exit depths indicated by the microdrive, and VRFs were estimated by projecting a recording site's relative location within a penetration onto the tectal map of visual space (Knudsen, 1982). Analysis was restricted to re- cording sites with VRFs located frontally, between $+20^{\circ}$ and $-30^{\circ}$ elevation and $\mathrm{L} 20^{\circ}$ and $\mathrm{R} 20^{\circ}$ azimuth. Within this region of space, ILDs of frequencies greater than $5 \mathrm{kHz}$ and ITDs vary almost exclusively and monotonically with sound source elevation and azimuth, respectively.

Auditory measurements. For each measurement, 10 series of stimuli were presented. Stimuli were sound bursts of $50 \mathrm{msec}$ duration, delivered at a rate of $1 / \mathrm{sec}$ and at an average binaural level of $20 \mathrm{~dB}$ above threshold. Within each series, ILD or ITD values were presented in randomized order. Net response to a stimulus was quantified by subtracting the number of spikes during a $100 \mathrm{msec}$ interval prior to stimulus presentation (spontaneous discharge rate) from the number of spikes 
The Journal of Neuroscience, September 1992, 12(9) 3477

LEFT EAR OCCLUSION
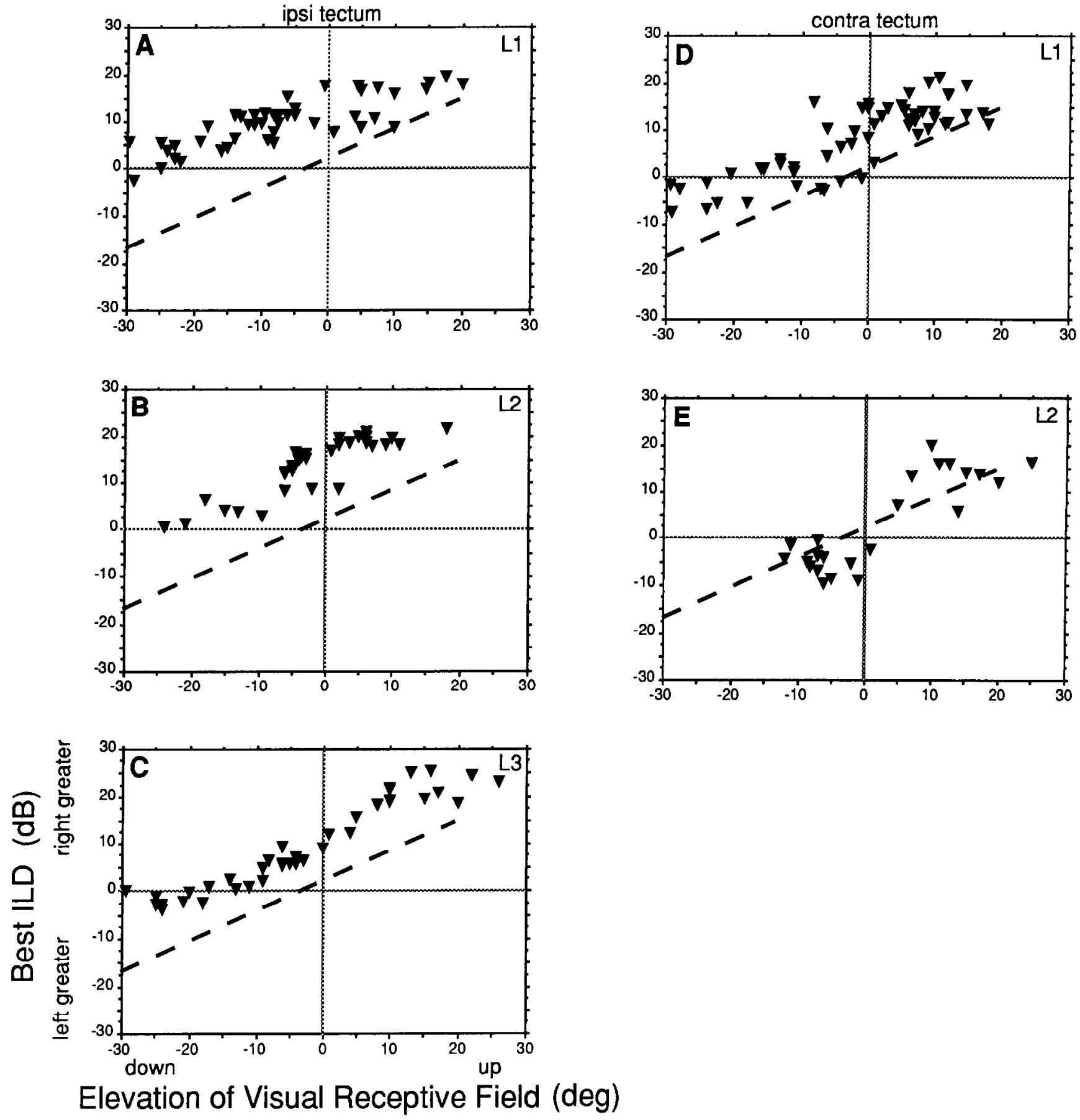

Figure 4. Relationship between best ILD and VRF elevation in individual testa of left-earplugged owls. $A-C$, Best ILLs in the testa ipsilateral to the occluded ear. $D$ and $E$, Best IDs in the tecta contralateral to the occluded ear. Recording sites with VRFs located above $+20^{\circ}$ elevation were not included in statistical analysis. For comparison, the regression line calculated for normal owls is shown as a broken line in each plot.

occurring during the 100 msec following the onset of the stimulus. Best ILD and best ITD were defined as the midpoint of the range of values for which a stimulus produced more than $50 \%$ of the maximum response. When ILD tuning was measured, ITD was held constant at its best value; when ITD tuning was measured, ILD was held constant at its best value.

Assessment of changes in unit tuning. Best ILDs and best IDs in monaurally occluded owls were compared with those recorded in normal owls. The normal regressions of best ILD on VRF elevation and of best ITD on VRF azimuth were calculated based on data from the 15 normal owls. Differences of best ILDs and best ITDs from earplugged owls from the normal regression lines were calculated. These values were compared with the residuals from the regression of data from the normal owls, using a one-tailed $t$-test to test for significance. The mean of the differences from the normal regression lines was used to represent the average change in unit tuning.

Results

Earplugs attenuate and delay sound arriving at the occluded ear (Knudsen et al., 1984). In order to align auditory best areas with VRFs, units must become tuned to the new values of binaural 

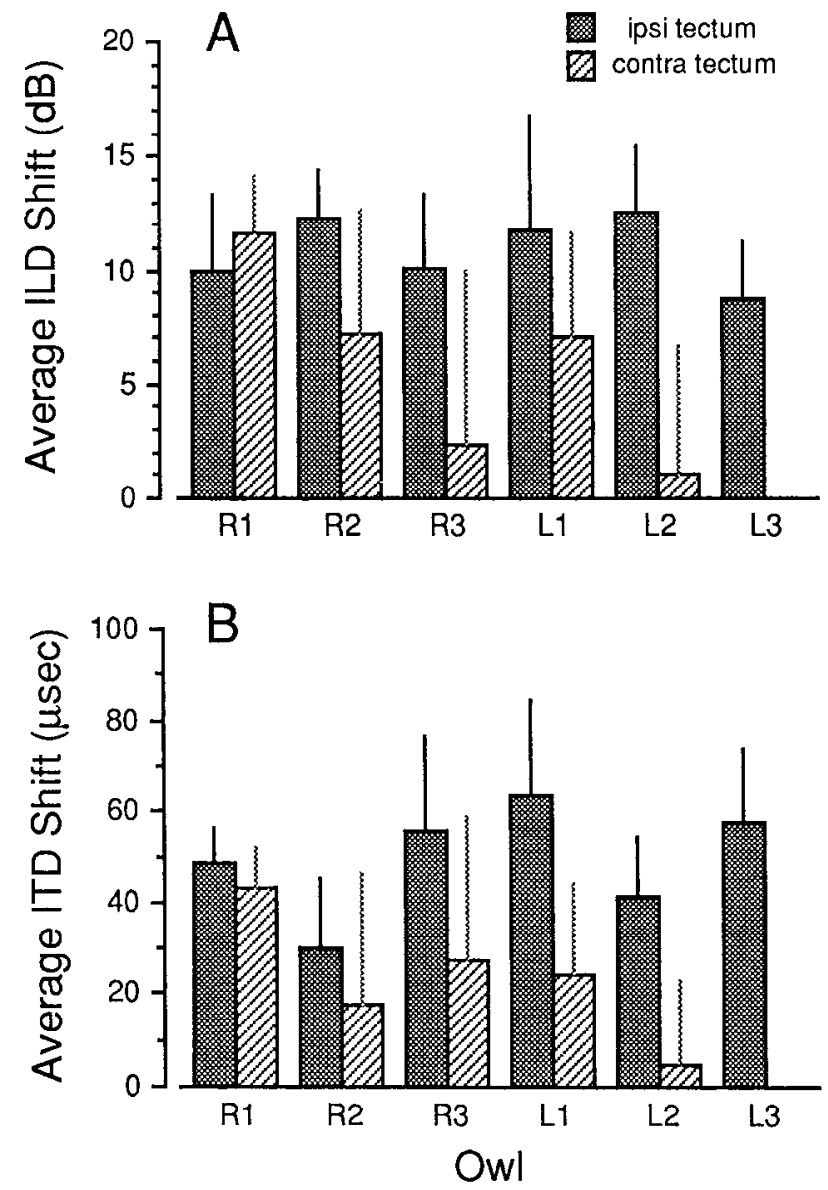

Figure 5. Average ILD shift $(A)$ and average ITD shift $(B)$ in the tectum ipsilateral and contralateral to the occluded ear in three right-earplugged owls $(R I-R 3)$ and three left-earplugged owls $(L 1-L 3)$. In owl $L 3$, data were gathered only from the ipsilateral tectum. The values are based on differences between measured best ILD or best ITD and the predicted values from the normal regression, as described in Figure 2. SDs (error bars) are large because the average shifts include data from both systematically shifted regions and regions of the tecta with nearly constant best values. The average shifts were, with the exception of the ILD shift in owl $R l$, larger in the ipsilateral than in the contralateral tectum $(p<$ $0.01)$.

cues corresponding to the locations of their VRFs. For a right ear occlusion, this would require shifts in unit tuning toward left-ear-greater ILDs and left-ear-leading ITDs, and for a left ear occlusion, it would require shifts in the opposite direction. Figure 1 compares the tuning to ILD and ITD of recording sites from a normal owl with those from a right- or a left-earplugged owl, all of the recording sites having similar VRF locations. In each case, the shift in tuning is in the adaptive direction, that is, in the direction of the untouched ear. These data exemplify the shifts in ILD and ITD tuning observed in the earplugged owls.

\section{Effects of monaural occlusion on ILD tuning}

A summary of best ILDs measured in normal and earplugged birds is shown in Figure 2. In normal owls, best ILD changed linearly with VRF elevation in the portion of the tectum that was examined (see Materials and Methods; Fig. $2 A$ ). Best ILD ranged from $18 \mathrm{~dB}$ right-ear-greater in the dorsal tectum to 15 $\mathrm{dB}$ left-ear-greater in the ventral tectum. In earplugged owls, best ILD also changed linearly with VRF elevation, but best ILDs were shifted in the adaptive direction (Fig. $2 C, E$ ). Best
ILDs in right-earplugged owls ranged from $8 \mathrm{~dB}$ right-ear-greater to $26 \mathrm{~dB}$ left-ear-greater, and in left-earplugged owls, they ranged from $26 \mathrm{~dB}$ right-ear-greater to $10 \mathrm{~dB}$ left-ear-greater. Thus, monaural occlusion resulted in best ILD values that exceded the range of $20 \mathrm{~dB}$ right/left-ear-greater measured in normal owls (Olsen et al., 1989). The average magnitude of the ILD shift (see Materials and Methods) was $8.9 \mathrm{~dB}$ toward left-eargreater for the three right-earplugged owls and $8.5 \mathrm{~dB}$ toward right-ear-greater for the three left-earplugged owls (Fig. 2D,F). A small number of recording sites with VRFs between $0^{\circ}$ and $-15^{\circ}$ elevation had best ILDs that were not shifted in the adaptive direction (Fig. 2C,E). These recording sites were in tecta that contained abnormal plateaus in the ILD map, as described below.

Monaural occlusion had two distinct effects on the map of best ILD: in some portions of the tectum, the map was shifted systematically; in others, the progression of best ILD plateaued. These two effects are most apparent in the data from individual tecta (Figs. 3, 4). The data from the right tectum of owl R3 (Fig. $3 C$ ) exhibited both effects. For recording sites with VRFs located below $+5^{\circ}$ elevation, best ILDs changed systematically with VRF elevation and were shifted toward left-ear-greater values by an average of $9.9 \mathrm{~dB}$. In contrast, for recording sites with VRFs located above $+5^{\circ}$, best ILDs remained nearly constant with VRF elevation, most units being tuned to ILDs near $0 \mathrm{~dB}$. Systematic shifts in the ILD map occurred in all of the tecta ipsilateral to the occluded ear (Figs. $3 A-C, 4 A-C$ ) and in two of the contralateral tecta (Figs. 3D, 4D). Plateauing of best ILD values was observed in the ipsilateral tectum of owl R3 (Fig. $3 C$ ), and in the contralateral tectum of owl R2 (Fig. $3 E$ ). Suggestions of similar plateaues in the ILD map were also observed in the contralateral tectum of owl R1 (Fig. 3D) and owl R3 (Fig. $3 F$ ) and in ipsilateral tectum of owl L3 (Fig. 4C).

The portion of the tectum that contained a shifted ILD map or best ILDs that were essentially constant was idiosyncratic. In one of the tecta (Fig. $3 A$ ), the ILD map was systematically shifted throughout the sampled region. In another (Fig. $3 E$ ), best ILDs remained near $0 \mathrm{~dB}$ across the entire sampled region. The extents of the systematically shifted versus plateaued portions of the ILD map could vary even between the tecta in a single animal. For example, the data from the left tectum of owl R3 (Fig. 3F) show best ILDs near $0 \mathrm{~dB}$ for recording sites with VRF elevations down to $-10^{\circ}$ on the left side, but only down to $+5^{\circ}$ on the right (Fig. 3C).

Plateauing of best ILD values occurred most frequently in the portion of the tectum that normally represented ILDs favoring the occluded ear. For right ear occlusions, this corresponds to recording sites with VRFs located above $0^{\circ}$ elevation, and for left ear occlusions, it corresponds to recording sites with VRFs located below $-10^{\circ}$ elevation (based on the normal regression shown in Fig. $2 A$ ). In two tecta (Fig. $3 E, F$ ), plateauing extended even beyond this portion of the tectum into regions that normally represent ILDs favoring the nonoccluded ear. The ILD value to which units were tuncd throughout the plateau region of the tectum was approximately $0 \mathrm{~dB}$ (Figs. $3 C, E, F ; 4 C$ ).

One tectum exhibited a relatively normal ILD map (Fig. $4 E$ ), even though the map on the opposite side was clearly shifted (Fig. 4B). The only abnormal aspect to this map was the weakness of the correlation of best ILD with VRF elevation $\left(r^{2}=\right.$ $0.72)$ relative to that observed in normal owls $\left(r^{2}=0.89\right)$.

Although the pattern of best ILD that resulted from monaural occlusion could be idiosyncratic, best ILDs tended to be shifted more systematically in the tectum ipsilateral to the occluded ear 

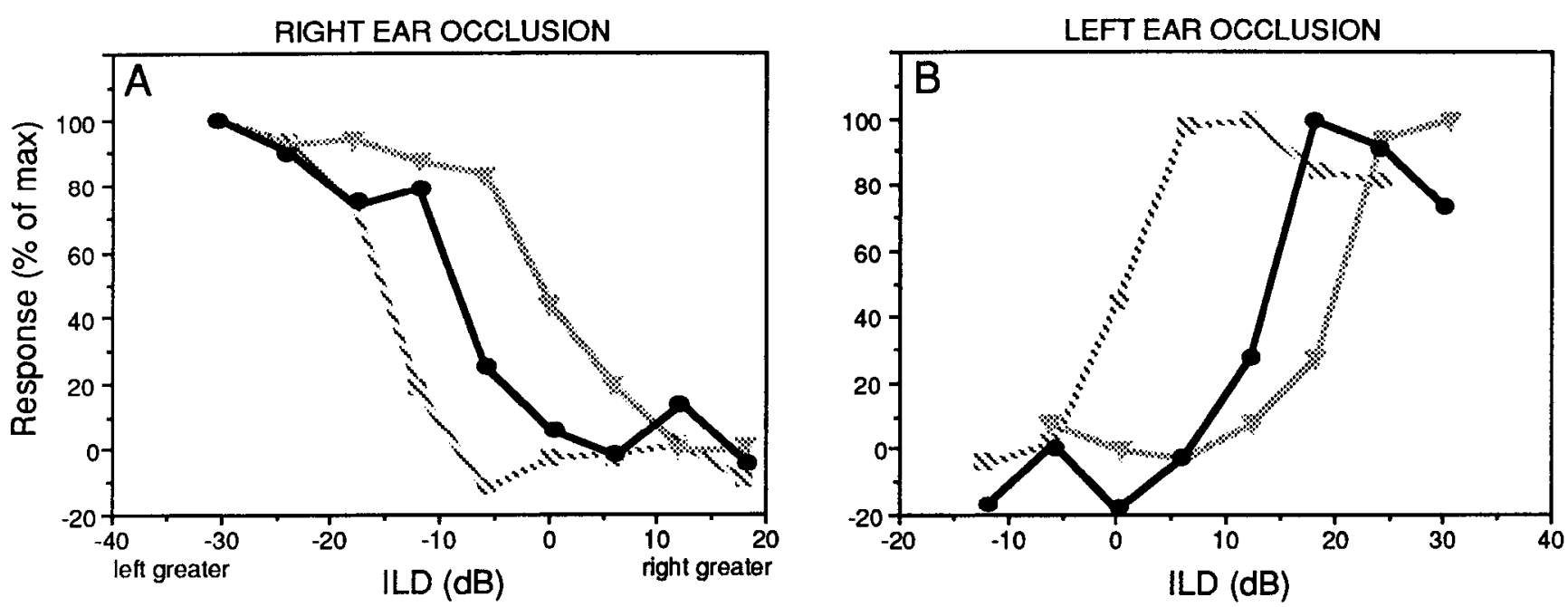

Figure 6. ILD tuning curves that are open to one side. The graphs show the range of open ILD tuning curves from owls raised with the right (A) or left $(B)$ ear occluded (Table 1). The tuning curves shown represent the leftmost (stippled lines), rightmost (hatched lines), and median (solid lines) cutoffs found in each owl.

(Figs. 3, 4). The average ILD shift (see Materials and Methods) calculated for each tectum individually was, with the exception of owl R1, larger in the ipsilateral than in the contralateral tectum $(p<0.01 ;$ Fig. $5 A)$.

Another abnormal aspect of ILD tuning in earplugged owls was the prevalence of recording sites that did not have closed tuning curves to ILD (also called excitatory-inhibitory, or EI, tuning curves). In the tectal representation of frontal space in normal owls only 3 out of 91 recording sites $(3.3 \%)$ had ILD tuning curves that were open to one side: two were open to rightear-greater and one to left-ear-greater. Among earplugged birds, 23 out of 419 recording sites $(5.5 \%)$ exhibited this property. However, in earplugged birds, the side of the tuning curve that was open depended absolutely on the ear that had been occluded (Fig. 6): in left-earplugged owls, all of the open curves were open toward right-ear-greater ILDs (and were found only among recording sites with VRFs located in upper space), and in rightearplugged owls, all of the open curves were open toward leftear-greater ILDs (and were found, with one exception, only among recording sites with VRFs located in lower space). Otherwise, ILD tuning widths were, for the most part, unaffected by monaural occlusion (Table 1 ).

\section{Effects of monaural occlusion on ITD tuning}

Monaural occlusion had no consistent effect on the sharpness of unit tuning to ITD (Table 1), but did systematically alter the value of ITD to which units were tuned. Figure 7 shows the best ITDs of all recording sites in normal and earplugged owls. In normal owls, best ITD changed linearly with VRF azimuth and ranged from $61 \mu \mathrm{sec}$ left-ear-leading to $49 \mu \mathrm{sec}$ right-earleading for recording sites with VRFs located between $\mathrm{L} 20^{\circ}$ and $\mathrm{R} 20^{\circ}$ (Fig. $7 A$ ). In earplugged owls, best ITD also changed linearly with VRF azimuth but best ITDs were shifted in the adaptive direction (Fig. $7 C, E$ ). In right-earplugged owls, best ITDs ranged from $137 \mu \mathrm{sec}$ left-ear-leading to $34 \mu \mathrm{sec}$ right-ear-leading, and in left-earplugged owls, they ranged from $56 \mu \mathrm{sec}$ leftear-leading to $117 \mu \mathrm{sec}$ right-ear-leading for recording sites in the same portion of the tectum. The average magnitude of the shift was $40 \mu \mathrm{sec}$ toward left-ear-leading for all right-earplugged owls and $40 \mu \mathrm{sec}$ toward right-ear-leading for all left-earplugged owls (Fig. $7 D, F$ ).
Considered individually, ITD maps were shifted in the adaptive direction in all of the tecta but one (Figs. 8, 9). In three of five owls, the average shift in best ITD was about twice as large on the ipsilateral side compared with the contralateral side (Fig. $5 B)$. In one owl, the shifts were approximately equal on the two sides (owl R1; Fig. $8 A, D$ ), and in another, best ITDs were shifted substantially (by $42 \mu \mathrm{sec}$ ) on one side, but only by $4 \mu \mathrm{sec}$ (unshifted) on the other (owl L2; Fig. 9B,E).

The largest average shift in best ITD was $63 \mu \mathrm{sec}$ in the left tectum of owl L1 (Fig. 9A). The tecta of this owl also exhibited the largest difference in the size of the average shift on the left and right sides: 63 versus $24 \mu \mathrm{sec}$, respectively. The tectum that

\begin{tabular}{|c|c|c|c|c|c|c|c|}
\hline & \multirow{2}{*}{$\begin{array}{l}\text { Nor- } \\
\text { mal }^{b}\end{array}$} & \multicolumn{3}{|c|}{ Right ear occluded } & \multicolumn{3}{|c|}{ Left ear occluded } \\
\hline & & $\mathrm{R} 1$ & R2 & R3 & $\mathrm{L} 1$ & $\mathrm{~L} 2$ & $\mathrm{~L}^{2}{ }^{c}$ \\
\hline \multicolumn{8}{|c|}{ ILD (50\% width, $\mathrm{dB}$ ) } \\
\hline Mean & 12.7 & 14.6 & 12.8 & 14.4 & $16.8^{*}$ & $10.2^{*}$ & 15.7 \\
\hline SD & 4.3 & 3.8 & 4.6 & 4.8 & 6.5 & 3.6 & 4.7 \\
\hline$n$ & $88^{d}$ & 96 & $35^{e}$ & $81^{r}$ & $95^{8}$ & $54^{h}$ & 35 \\
\hline \multicolumn{8}{|c|}{ ITD ( $50 \%$ width, $\mu \mathrm{sec})$} \\
\hline Mean & 43.2 & 43.4 & $58.6^{*}$ & 49.1 & $50.9^{*}$ & 43.5 & 38.5 \\
\hline SD & 14.9 & 14.9 & 19.0 & 15.6 & 19.1 & 12.8 & 14.0 \\
\hline$n$ & 91 & 96 & 39 & 89 & 98 & 56 & 36 \\
\hline
\end{tabular}

a All recording sites with visual receptive fields were centered between $\mathrm{L} 20^{\circ}$ and $\mathrm{R} 20^{\circ}$ and between $-30^{\circ}$ and $+20^{\circ}$

${ }^{b}$ Data from 15 owls.

C Data from left tectum only.

${ }^{d}$ Three recording sites had ILD tuning curves that were open to one side; two were open to right-ear-greater, one was open to left-ear-greater. These were not included in the mean.

${ }^{C}$ Four recording sites had ILD tuning curves that were open to left-ear-greater; these were not included in the mean.

Nine recording sites had ILD tuning curves that were open to left-ear-greater; these were not included in the mean.

${ }^{z}$ Eight recording sites had ILD tuning curves that were open to right-ear-greater; these were not included in the mean.

${ }^{h}$ Two recording sites had ILD tuning curves that were open to right-ear-greater; these were not included in the mean.

* Significantly different from normal $(p<0.001)$. 

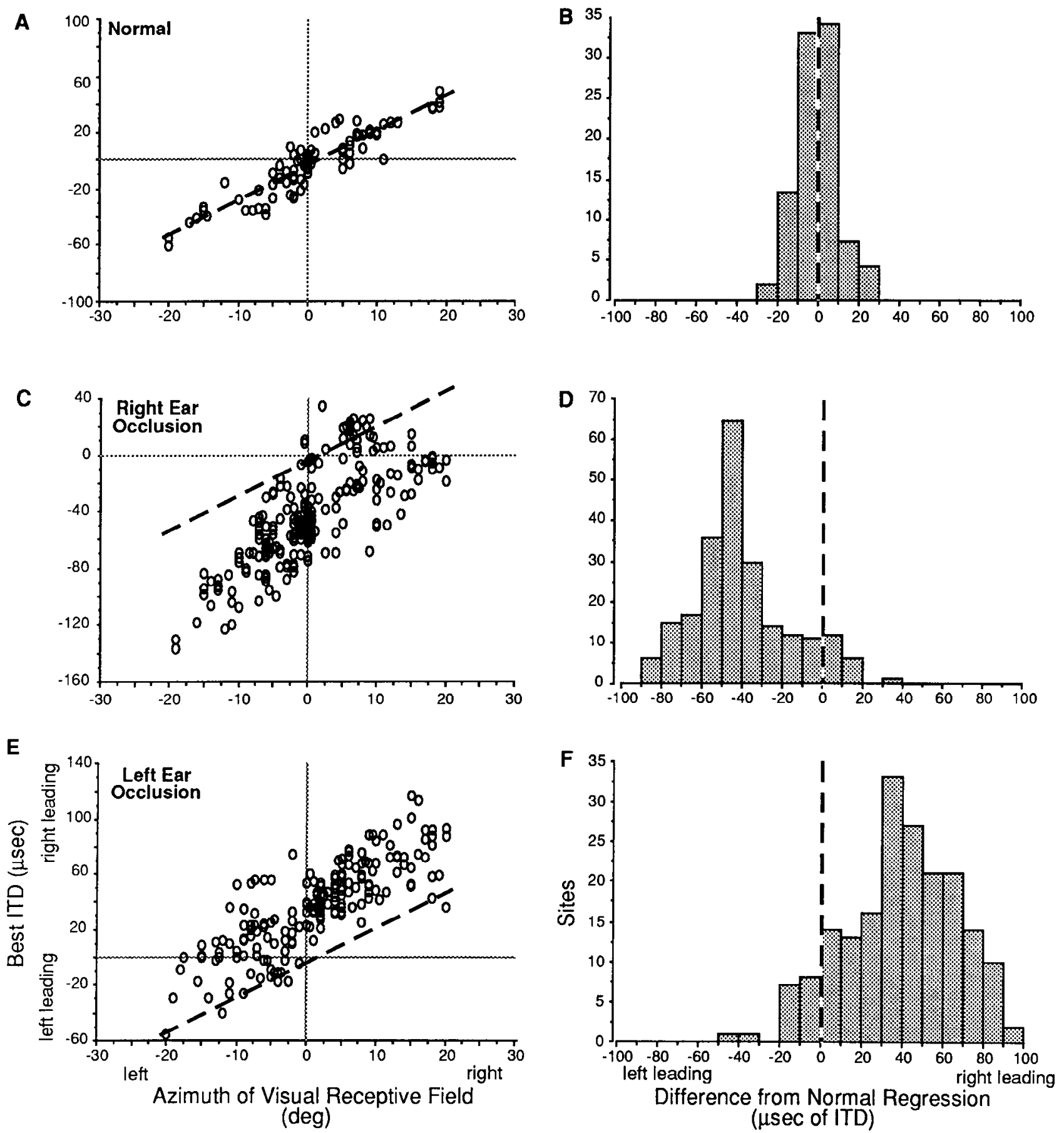

Figure 7. Relationship between best ITD and VRF azimuth. Data are restricted to recording sites with VRFs located between $+20^{\circ}$ and $-30^{\circ}$ elevation and between $\mathbf{L} 20^{\circ}$ and $\mathrm{R} 20^{\circ}$ azimuth. $A$, Results of 91 recording sites from 15 normal owls. The regression line $(b r o k e n ~ l i n e$ in $A, C$, $E$; $y=2.49 x-3.94 ; r^{2}=0.82$ ) was calculated to assess shifts in best ITDs measured in earplugged owls. $C$, Results of 224 recording sites from three right-earplugged owls. The regression for this data set $\left(y=3.28 x-44.9 ; r^{2}=0.57\right)$ was shifted toward left-ear-leading ITDs. $E$, Results of 190 recording sites from three left-earplugged owls. The regression for this data set $\left(y=2.79 x+33.06 ; r^{2}=0.65\right)$ was shifted to right-ear-leading ITDs. $B$, Differences between best ITDs measured in normal owls $(A)$ and best ITDs predicted from the normal regression line. $D$, Differences between best ITDs measured in right-earplugged owls $(C)$ and best ITD predicted from the normal regression line (mean, $40.3 \mu$ sec left-ear-leading; significantly different from $0, p<0.001)$. $F$, Differences between best ITDs measured in left-earplugged owls $(E)$ and best ITD predicted from the normal regression line (mean, $40.3 \mu \mathrm{sec}$ right-ear-leading; significantly different from $0, p<0.001$ ). The data for each owl are plotted separately in Figures 9 and 10 .

exhibited normal ITD tuning (right tectum, owl L2; Fig. 9E) was the same tectum that exhibited essentially normal ILD tuning (Fig. $4 E$ ). Surprisingly, both the ITD and ILD maps were clearly shifted in the tectum on the other side in this owl (Figs. $4 B, 9 B)$.

\section{Discussion}

Effects of monaural occlusion on unit tuning to ILD and ITD The results demonstrate that in barn owls raised with one ear occluded, the tuning of tectal units to ILD and ITD is altered 


\section{RIGHT EAR OCCLUSION}
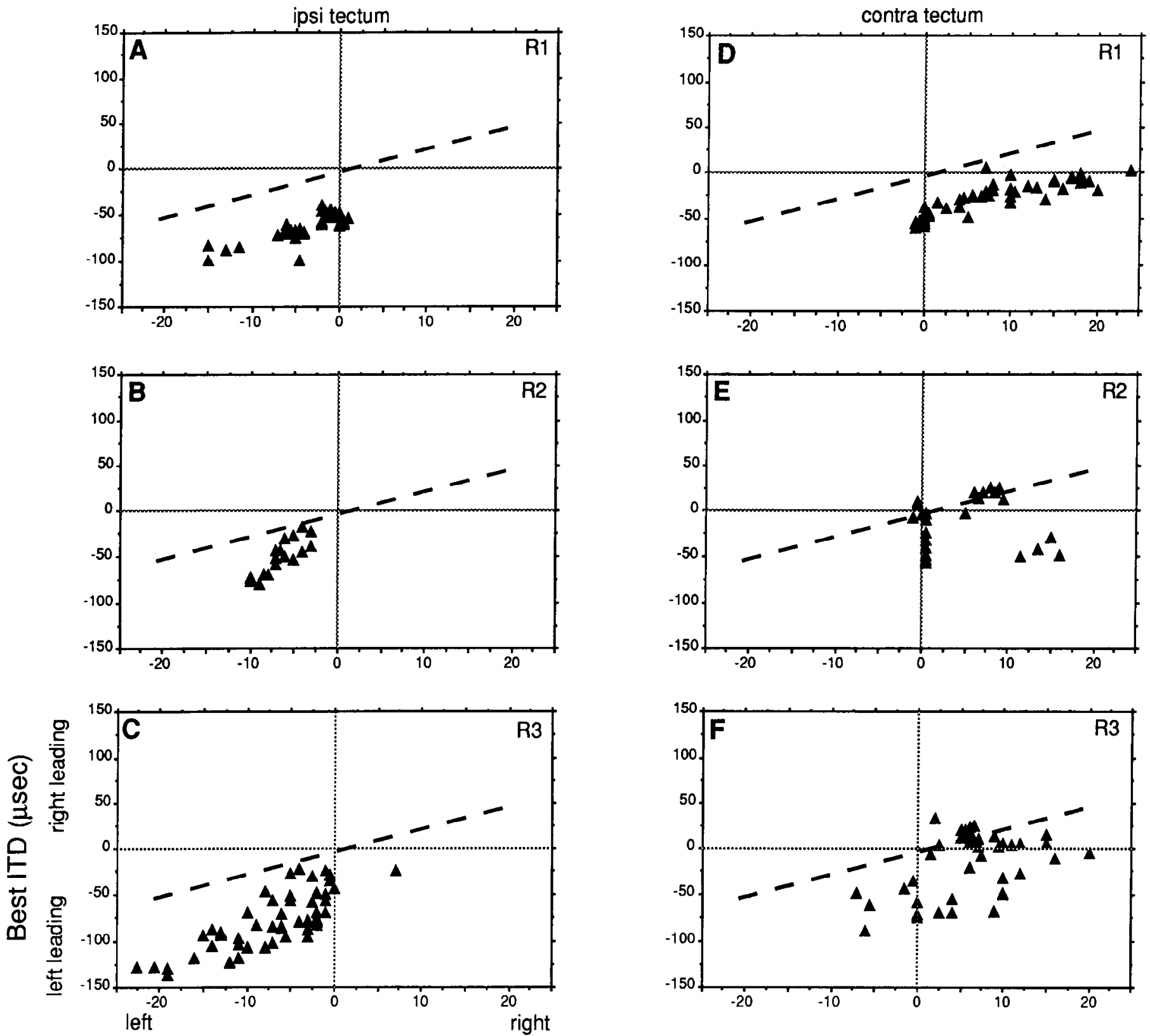

Azimuth of Visual Receptive Field (deg)

Figure 8. Relationship between best ITD and VRF azimuth in individual tecta of right-earplugged owls. $A-C$, Best ITDs in the tecta ipsilateral to the occluded ear. $D-F$, Best ITDs in the tecta contralateral to the occluded ear. For comparison, the regression line calculated for normal owls is shown as a broken line in each plot.

in directions that compensate for the effects of the occlusion. The magnitude of the median average shift across the 11 sampled tecta was $9.9 \mathrm{~dB}$ for best ILD and $41.5 \mu \mathrm{sec}$ for best ITD (Fig. 5); note that the average shift calculated for individual tecta includes data both from the systematically shifted portions of the ILD map and from regions of the tectum where best ILDs were nearly constant. ILD and ITD shifts of this size translate into spatial shifts of about $20^{\circ}$ in elevation and $15^{\circ}$ in azimuth, respectively, across frontal space for a one-third octave band of frequencies centered on $7 \mathrm{kHz}$ (Knudsen et al., 1991), a frequency range to which most units in the rostral tectum respond (Knudsen, 1984). Previous studies in the barn owl have shown that early monaural occlusion results in adjustment of auditory spatial tuning of tectal units (Knudsen, 1985) and adjustments in sound localization behavior (Knudsen et al., 1984; Knudsen, 1985) of about this magnitude. Thus, the adjustments reported here are appropriate to account for the adaptive adjustment of auditory spatial tuning and sound localization behavior reported previously.

\section{Variability of effects across tecta}

Although monaural occlusion caused adaptive shifts in unit tuning in both optic tecta, the shift tended to be larger and more systematic in the tectum ipsilateral to the occluded ear. The 


\section{LEFT EAR OCCLUSION}
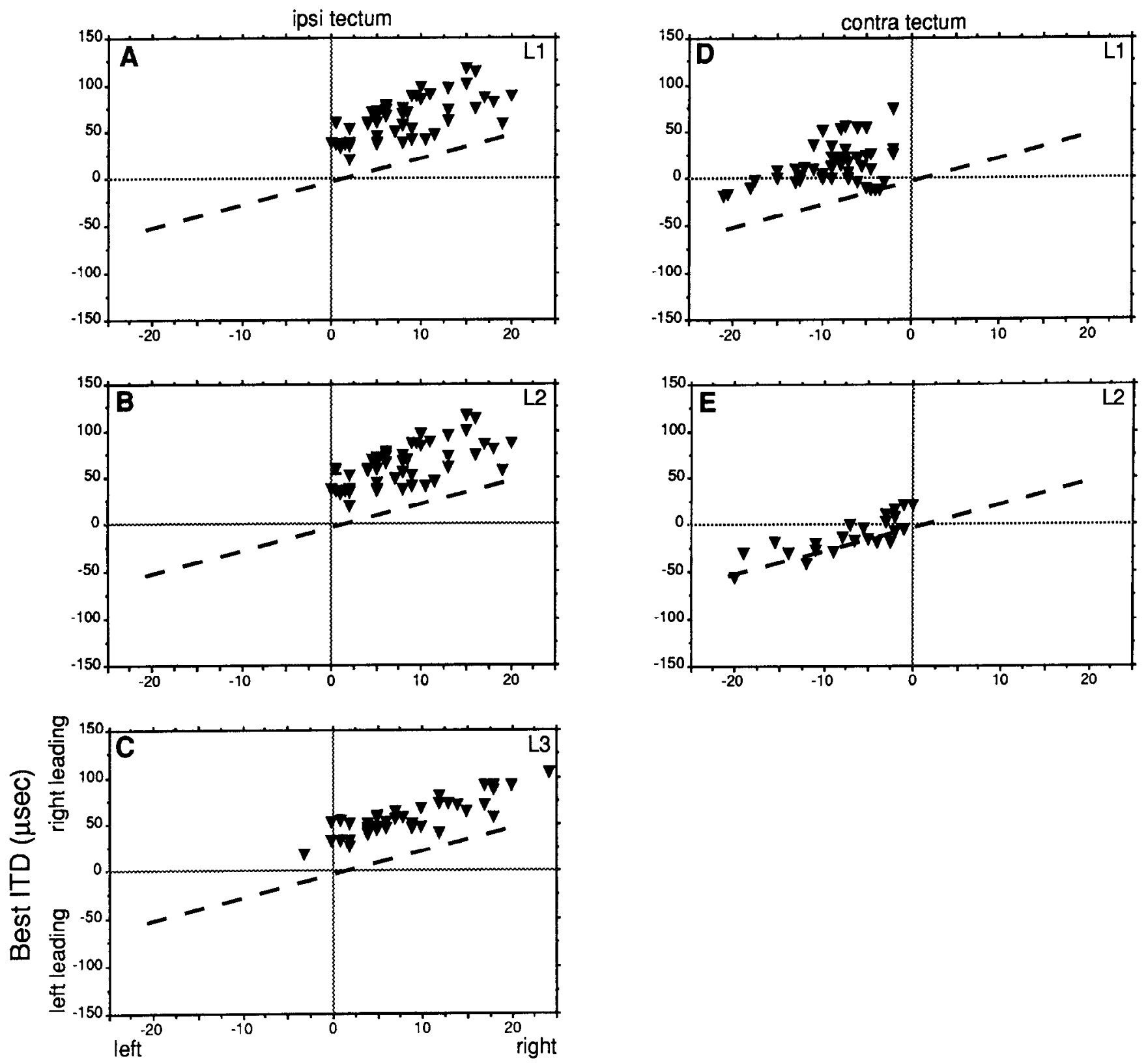

Azimuth of Visual Receptive Field (deg)

Figure 9. Relationship between best ITD and VRF azimuth in individual tecta of left-earplugged owls. $A-C$, Best ITDs in the tecta ipsilateral to the occluded ear. $D$ and $E$, Best ITDs in the tecta contralateral to the occluded ear. For comparison, the regression line calculated for normal owls is shown as a broken line in each plot.

average magnitude of the ILD shift ranged from 8.7 to $12.5 \mathrm{~dB}$ (median, $11.0 \mathrm{~dB}$ ), the variation in magnitude reflecting, at least in part, variability in the effectiveness of different earplugs (Knudsen et al., 1984). The average magnitude of the ITD shift ranged from 30 to $63 \mu \mathrm{sec}$ (median, $47 \mu \mathrm{sec}$ ). Adaptive adjustments in the contralateral tectum ranged from 1.1 to $11.6 \mathrm{~dB}$ (median, $7.0 \mathrm{~dB}$ ) for ILD and from 5 to $43 \mu \mathrm{sec}$ (median, 24 $\mu \mathrm{sec})$ for ITD. The cause of this asymmetry in the magnitude of the effect on the two sides of the brain is not apparent.

In addition to (or instead of) systematic adjustments of ILD tuning, apparent plateauing of the progression of best ILD values across the tectum was observed in at least two of the six owls
(Fig. $3 C, E$ ). This plateauing of the ILD map occurred in the portion of the tectum where units normally are tuned to ILDs favoring the occluded ear. Moreover, in each case, the value of ILD to which units were tuned in the plateau region was approximately $0 \mathrm{~dB}$. Consequently, in this part of the map, the deviations of best ILDs from their predicted values based on the normal regression could be exceptionally large, up to $20 \mathrm{~dB}$. However, ILD plateaus were unpredictable: plateauing in one tectum did not necessarily mean plateauing in the tectum on the opposite side (Fig. 3, $B$ vs. E); and when plateauing occurred on both sides, it could vary greatly in extent (Fig. 3, $C$ vs. $F$ ). Thus, although the direction of the ILD shift of units in the 
plateau regions was usually in the adaptive direction, the plateau itself may reflect a process that is not necessarily an adaptive process. Our current understanding of how ILD information is processed by the auditory system provides no explanation for how or why monaural occlusion causes plateaus in the ILD map.

\section{Sites of adjustment}

It is possible that monaural occlusion affects ILD and ITD processing at various levels in the ascending auditory pathway. Adjustments in the ILD and ITD maps that are equivalent in the left and right tecta could be explained by an experiencedependent regulation of the monaural signals. The instructional influence could be based on information intrinsic to the auditory system. For example, the brain might regulate the gains of the monaural signals so that they carry approximately equivalent ranges of discharge rates when averaged over days or weeks. Also, the brain might adjust the phase response of the monaural signals so that the range of phase differences experienced over days or weeks is symmetrical. Note that these kinds of adjustments could be made without access to visual or other extrinsic sources of spatial information (Knudsen and Brainard, 1991; Knudsen and Mogdans, 1992).

In contrast, adjustments that are not equivalent in the left and right tecta cannot be accounted for by the regulation of monaural gains and phase responses. Nonequivalent adjustments include large differences in the magnitude of the adjustments in the ILD and ITD maps (Fig. 5) and in the topographies of the altered ILD maps on the left and right sides (Figs. 3, 4). Nonequivalent adjustments must reflect effects of monaural occlusion that influence ILD and ITD processing at or beyond the site where the encoding of left and right space separate in the auditory pathway.

On the other hand, adjustment early in the binaural pathway is suggested by the results of an anatomical study in which HRP was injected into the tectum of an owl with a permanently altered auditory space map (Knudsen, 1985). The positions of retrogradely labeled cells in the external nucleus of the inferior colliculus (ICX) were the same as those observed in normal owls. This shows that occlusion-induced changes in the auditory space map in the optic tectum are not due to changes in the anatomical projection from the ICx and implies that the experience-dependent adjustments take place at or before the $\mathrm{ICx}$. This result is consistent with data from a study in which chronic monaural occlusion of kittens was shown to alter the ILD sensitivity of units in the central nucleus of the IC (Moore and Irvine, 1981). In that study, the strength of the inhibitory influence from the nonoccluded ear on contralateral IC units was found to be decreased, the same direction of effect as reported here for owls. A study on rats also describes changes in the ILD sensitivity of IC units as a result of rearing animals with a monaural occlusion, although in this case the effect was in the opposite direction (Silverman and Clopton, 1977).

\section{Experience-dependent aspects of the map}

The auditory space map is exceptionally plastic during development. First, experience with monaural occlusion or displacing prisms can result in the representation of ITD and ILD values that normally are not represented in the map (Knudsen and Brainard, 1991; Figs. 3, 4, 8, 9). The range of Best ITDs, limited normally to $25 \mu \mathrm{sec}$ ipsi-ear-leading at the rostral boarder of the map (Olsen et al., 1989), could extend to $90 \mu \mathrm{sec}$ ipsi-earleading in earplugged owls (Fig. $8 F$ ). Similarly, the range of best
ILDs, which is limited to values of less than $20 \mathrm{~dB}$ in normal owls (Olsen et al., 1989), could extend to values of up to $26 \mathrm{~dB}$ in earplugged owls (Figs. 3C, 4C). Thus, the ranges of best ITDs and best ILDs that come to be represented in the map are not prespecified but are selected from wider possible ranges based on experience. Second, the pattern of progression, at least of best ILDs, can be altered dramatically by monaural occlusion in such a way that in some portions of the tectum best ILDs change with VRF elevation while in others they remain at a plateau value. Also, raising owls with the sound-collecting surfaces of the external ears (facial ruff feathers) removed alters dramatically the rate at which best ILDs and best ITDs vary across the tectum (Olsen and Knudsen, 1989). Third, the orientation of the space map can be flipped upside down along the elevational dimension, as a result of raising owls without vision (Knudsen et al., 1991). As shown in the following companion article (Knudsen and Mogdans, 1992), the visual spccification of the auditory map's orientation does not occur until sometime after 1 month of age. Finally, in guinea pigs, the very existence of an auditory space map in the optic tectum seems to require both visual and auditory experience with localizable sources during early development (Withington-Wray et al., 1990a,b).

The correspondence between the values of sound localization cues and source locations in space varies across individuals (Knudsen et al., 1991). Moreover, during development this correspondence changes as the head and ears grow. The plasticity of auditory information processing, described above, enables the creation of a precise, veridical map of space by allowing experience to specify the exact cue-location associations that are appropriate for the individual.

\section{References}

Brainard MS, Knudsen EI, Esterly SD (1992) Neural derivation of sound source location: resolution of spatial ambiguities in binaural cues. J Acoust Soc Am 91:1015-1027.

King AJ, Hutchings ME (1987) Spatial response properties of acoustically responsive neurons in the superior colliculus of the ferret: a map of auditory space. J Neurophysiol 57:596-624.

King AJ, Palmer AR (1983) Cells responsive to free-field auditory stimuli in guinea-pig superior colliculus: distribution and response properties. J Physiol (Lond) 342:361-381.

King AJ, Hutchings ME, Moore DR, Blakemore C (1988) Developmental plasticity in the visual and auditory representations in the mammalian superior colliculus. Nature 332:73-76.

Knudsen EI (1982) Auditory and visual maps of space in the optic tectum of the owl. J Neurosci 2:1177-1194.

Knudsen EI (1983) Early auditory experience aligns the auditory map of space in the optic tectum of the barn owl. Science 222:939-942.

Knudsen EI (1984) Auditory properties of space-luned units in the owl's optic tectum. J Neurophysiol 52:709-723.

Knudsen EI (1985) Experience alters the spatial tuning of auditory units in the optic tectum during a sensitive period in the barn owl. J Neurosci 5:3094-3109.

Knudsen EI, Brainard MS (1991) Visual instruction of the neural map of auditory space in the developing optic tectum. Science 253:85-87.

Knudsen EI, Konishi M (1980) Monaural occlusion shifts the receptive field locations of auditory midbrain units in the owl. J Neurophysiol 44:687-695.

Knudsen EI, Mogdans J (1992) Vision-dependent adjustment of unit tuning to sound localization cues in response to monaural occlusion in developing owl optic tectum. J Neurosci 12:3485-3493.

Knudsen EI, Esterly SD, Knudsen PF (1984) Monaural occlusion alters sound localization during a sensitive period in the barn owl. J Neurosci 4:1001-1011.

Knudsen EI, Esterly SD, du Lac S (1991) Stretched and upside-down maps of auditory space in the optic tectum of blind-reared owls; acoustic basis and behavioral correlates. J Neurosci 11:1727-1747. 
Middlebrooks JC, Knudsen EI (1984) A neural code for auditory space in the cat's superior colliculus. J Neurophysiol 4:2621-2634.

Moiseff A, Konishi M (1981) The owl's interaural pathway is not involved in sound localization. J Comp Physiol 144:299-304.

Moore DR, Irvine DR (1981) Plasticity of binaural interaction in the cat inferior colliculus. Brain Res 208:198-202.

Olsen JF, Knudsen EI (1989) Adaptive changes in binaural tuning of neurons in the optic tectum of barn owls raised with altered external ears. Soc Neurosci Abstr 15:290.

Olsen JF, Knudsen EI, Esterly SD (1989) Neural maps of interaural time and intensity differences in the optic tectum of the barn owl. J Ncurosci 9:2591-2605.
Silverman MS, Cloptun BM (1977) Plasticity of binaural interaction. I. Effect of early auditory deprivation. J Neurophysiol 40:1266-1274.

Withington-Wray DJ, Binns KE, Keating MJ (1990a) The maturation of the superior collicular map of auditory space in the guinea pig is disrupted by developmental visual deprivation. Eur J Neurosci 2: 683-692.

Withington-Wray DJ, Binns KE, Dhanjal SS, Brickley SG, Keating MJ (1990b) The maturation of the superior collicular map of auditory space in the guinea pig is disrupted by developmental auditory deprivation. Eur J Neurosci 2:693-703. 